\title{
Prevalência, tratamento e controle da hipertensão arterial com método diferenciado de busca ativa
}

\section{Prevalence, treatment and control of hypertension with a differential method of active search}

\author{
Thiago de Castro Menezes ${ }^{1}$, Leslie Andrews Portes ${ }^{1,2}$, Natália Cristina de Oliveira Vargas e Silva' (D) \\ ' Programa de Mestrado em Promoção da Saúde, Centro Universitário Adventista de São Paulo (UNASP) - São Paulo (SP), Brasil. \\ ${ }^{2}$ Laboratório de Fisiologia do Exercício (LAFEX), Centro Universitário Adventista de São Paulo (UNASP) - São Paulo (SP), Brasil.
}

Como citar: Menezes TC, Portes LA, Vargas e Silva NCO. Prevalência, tratamento e controle da hipertensão arterial com método diferenciado de busca ativa. Cad Saúde Colet, 2020;28(3):325-333. https://doi.org/10.1590/1414-462X202028030357

\section{Resumo}

Introdução: A hipertensão arterial sistêmica (HAS) atinge cerca de $30 \%$ da população adulta, sendo o maior fator de risco para lesões cardíacas e cerebrovasculares, e a terceira causa de invalidez. Objetivo: Determinar a prevalência de indivíduos com HAS e os níveis de conhecimento, tratamento e controle da doença, por meio de um método diferenciado de busca ativa. Método: Realizou-se estudo transversal, de base populacional, com amostra representativa composta por 409 indivíduos adultos, na área de abrangência de uma Unidade Básica de Saúde com Estratégia de Saúde da Família, na periferia da zona sul da cidade de São Paulo. As visitas domiciliares compreenderam medidas antropométricas e de pressão arterial (PA), além da aplicação de um questionário sociodemográfico. Resultados: A prevalência de HAS na população foi de 42,5\%. A maior parte dos indivíduos hipertensos (94\%) tinha conhecimento da doença, $95 \%$ deles faziam tratamento e $48 \%$ apresentavam PA controlada. Foram identificados 45 novos casos de HAS, desses, 77\% foram encontrados em horário diferenciado (domingos das 10 às $16 \mathrm{~h}$ ), representando $27 \%$ dos hipertensos. Conclusão: $O$ método diferenciado mostrou-se eficiente como estratégia de busca ativa para novos casos de HAS em locais onde ainda existe aparente subnotificação. Palavras-chave: prevalência; hipertensão; estilo de vida; saúde da família.

\begin{abstract}
Background: Systemic arterial hypertension (SAH) affects more than $30 \%$ of the adult population and is a major risk factor for heart and cerebrovascular injuries, and the third cause of disability. Objective:To determine the prevalence of individuals with hypertension and levels of awareness, treatment, and control of the disease, through a differential method of active search. Method: A cross-sectional population-based study was carried out with a representative sample of 409 adult individuals, in the area covered by a Basic Health Unit with Family Health Strategy, in the suburb of the southern area of São Paulo. Home visits included anthropometric and blood pressure (BP) measures, and the application of a sociodemographic questionnaire. Results: The prevalence of SAH was 42,5\%. Most of the hypertensive individuals (94\%) were aware of the disease, $95 \%$ of them were treated and $48 \%$ had the BP under control. Forty-five new cases of hypertension were identified, of which $77 \%$ were found in the differential schedule (Sundays from 10 am to $4 \mathrm{pm}$ ), representing 27\% of hypertensive patients. Conclusion: The differential method proved to be efficient as an active search strategy for new cases of hypertension in places where there is an apparent underreporting of this disease.
\end{abstract}

Keywords: prevalence; hypertension; lifestyle; family health.

Este é um artigo publicado em acesso aberto (Open Access) sob a licença Creative Commons Attribution, que permite uso distribuição e reprodução em qualquer meio, sem restrições desde que o trabalho original seja corretamente citado.

\footnotetext{
Trabalho realizado na atenção básica à saúde da região do Capão Redondo, São Paulo - SP, no segundo semestre de 2015. Correspondência: Natália Cristina de Oliveira Vargas e Silva. E-mail. natalia.silva@ucb.org.br

Fonte de Financiamento: nenhuma.

Conflito de interesses: nada a declarar.

Recebido em: Fev. 05, 2018. Aprovado em: Ago. 17, 2019
} 


\section{INTRODUÇÃO}

A hipertensão arterial sistêmica (HAS) é multifatorial e se caracteriza por níveis elevados e sustentados de pressão arterial (PA), cujos principais fatores de risco incluem idade, etnia, excesso de peso, ingestão de sódio, ingestão de álcool, sedentarismo, baixa escolaridade e fatores genéticos' ${ }^{1}$.

Cerca de $31 \%$ da população adulta, aproximadamente 1,2 bilhão de pessoas no mundo, é portadora de $\mathrm{HAS}^{2}$. No Brasil, a doença afeta cerca de $30 \%$ da população ${ }^{3}$ e constitui-se no maior fator de risco para lesões cardíacas e cerebrovasculares, além de ser importante causa de invalidez permanente ${ }^{4}$. Sua prevalência aumentou $8 \%$ de 2000 a 2010 em países como o Brasil, o que faz dela um dos mais importantes problemas de saúde pública da atualidade.

A despeito dos critérios bem definidos para seu tratamento medicamentoso e apesar da coexistência de estratégias de controle não farmacológicas, os índices de controle ainda são baixos ${ }^{2}$. Apenas um terço dos hipertensos brasileiros apresenta controle satisfatório da doença ${ }^{5}$, o que faz da HAS assunto complexo, pois envolve o custo dos medicamentos, a necessidade de se combinar mais de uma droga, os efeitos colaterais, a baixa adesão ao tratamento e as dificuldades relacionadas ao acesso e uso dos serviços de saúde ${ }^{6}$.

A Estratégia de Saúde da Família (ESF), criada em 1994, é a vertente brasileira da atenção primária à saúde (APS), caracterizada como a "porta de entrada" prioritária em um sistema de saúde constitucionalmente fundado no direito à saúde e na equidade do cuidado 7 . Com relação à HAS, a ESF se utiliza de várias ações que incluem medida da PA, consultas médicas e de enfermagem, atividades educativas individuais e coletivas, e acesso ao tratamento apropriado nos demais níveis hierárquicos do Sistema Único de Saúde brasileiro (SUS). As equipes da ESF trabalham com o intuito de identificar precocemente os portadores de HAS, acompanhá-los adequadamente, orientá-los sobre a manutenção dos parâmetros pressóricos em níveis normais e os riscos decorrentes da sua elevação ${ }^{8}$.

No ano de 2015, a cidade de São Paulo (SP, Brasil) contava com 1.124 equipes de ESF implantadas em 271 Unidades Básicas de Saúde (UBS), cobrindo 34\% da cidade. As equipes distribuíam-se preferencialmente nas regiões mais periféricas e/ou de alta vulnerabilidade, segundo avaliação das necessidades de saúde 9 .

A região do Capão Redondo conta com $100 \%$ de cobertura de ESF desde o ano de $2007^{10}$. Está localizada a cerca de $18 \mathrm{~km}$ do marco zero da cidade e possui índice de desenvolvimento humano de 0,782 , acima da média brasileira $(0,718)$, e renda per capita média de $\mathrm{R} \$ 1.034,82$ ( € 280 ou US\$ 300) ${ }^{11}$. O Capão Redondo é considerado uma região dormitório, porque grande proporção de seus residentes passa a maior parte do dia nas áreas centrais da cidade, onde existem mais oportunidades de emprego ${ }^{12}$. Nos 7 anos anteriores a este estudo, mais de 30.000 visitas domiciliares foram realizadas pelos profissionais da ESF na região ${ }^{10}$, e não houve aumento importante de novos casos de HAS. Em 2009, a prevalência da doença na população acima de 20 anos de idade era de 16\%, passou para $17 \%$ em 2010 e em 2011 , $18 \%$ em 2012 e 2013, 18,5\% em 2014 e, finalmente, em 2015 a prevalência de HAS observada foi de $19 \%$. Esse valor encontra-se abaixo da prevalência nacional $(30 \%)^{3}$ e da região sudeste do país $(22,8 \%)$, onde se encontra o estado de São Paulo ${ }^{13}$.

Diante da grande diferença verificada entre as prevalências, justificam-se novas avaliações, buscando identificar possíveis explicações. Assim, o objetivo deste estudo foi determinar a prevalência de HAS e os níveis de conhecimento, tratamento e controle da doença, por meio de um método diferenciado de busca de novos casos em uma população pertencente à área de abrangência de uma UBS da região do Capão Redondo (zona sul da cidade de São Paulo, Brasil).

\section{MÉTODO}

Trata-se de um estudo observacional, analítico, de delineamento transversal, de base populacional, com amostragem aleatória com reposição em múltiplos estágios. A UBS da região do Capão Redondo (zona sul da cidade de São Paulo, Brasil) abrange uma área de 3,2km², população de 25.857 pessoas (7.664 famílias), com atual prevalência de HAS de 18,59\% entre as pessoas maiores de 20 anos de idade ${ }^{10}$. $O$ tamanho amostral foi calculado como proposto 
por Kearney et al. ${ }^{14}$, considerando-se uma população de 18.559 habitantes maiores de 20 anos e um erro tolerável de 5\%, totalizando 392 indivíduos para uma amostra representativa.

A amostragem foi realizada em três estágios: 1- delineamento das 48 microáreas que cobriam o território pesquisado; 2- sorteio de 10 domicílios de cada microárea, identificando-se as 10 famílias; 3- sorteio de um morador de cada domicílio. Foram excluídas da amostra as gestantes.

A primeira tentativa de encontrar cada participante ocorreu no horário e período regular (HR) de funcionamento da UBS (de segunda a sexta-feira, das $7 \mathrm{~h}$ às $17 \mathrm{~h}$ ). Caso o indivíduo não estivesse no domicílio naquele momento, uma segunda tentativa era realizada, em período e horário diferenciados (HD): aos domingos, das 10 às 16h. Esse foi o método diferenciado do presente estudo. Após a segunda tentativa sem sucesso, ou em caso de recusa, era realizado um novo sorteio de outro indivíduo em domicílio contíguo, identificado no sentido horário.

O presente estudo foi aprovado pelos Comitês de Ética em pesquisa institucional e da Secretaria Municipal de Saúde da cidade de São Paulo. Todos os participantes forneceram consentimento escrito à participação na pesquisa.

Os participantes responderam a um questionário sociodemográfico que continha questões sobre o tratamento e o diagnóstico de HAS, confirmado pela apresentação de medicação anti-hipertensiva e registro desse dado no prontuário do paciente junto à UBS. Registrou-se também se a PA estava ( $\leq 140 / 90 \mathrm{mmHg}$ ) ou não controlada $(>140 / 90 \mathrm{mmHg})$. A coleta de dados ocorreu entre os meses de abril e outubro de 2015, e os novos casos de HAS identificados foram encaminhados para tratamento na UBS de referência. Foram considerados novos casos de HAS os pacientes diagnosticados por este estudo que não sabiam serem portadores desta condição, quando esta informação também não constasse de seu prontuário na UBS. Os casos antigos considerados foram aqueles cujo diagnóstico já havia sido feito pelos profissionais na UBS previamente à realização do estudo, e que haviam sido registrados no prontuário dos pacientes.

A medida da PA foi feita com esfigmomanômetro automático (Omron HEM790it) com o indivíduo na posição sentada, pés no chão, braço esquerdo relaxado, apoiado sobre mesa e à altura do coração, palma da mão voltada para cima, bexiga vazia, na ausência de prática de exercícios físicos moderados ou intensos nas últimas 2 horas, e sem que o indivíduo tivesse fumado ou ingerido bebida alcoólica nos 30 minutos antecedentes.

Conforme critérios diagnósticos de HAS', foram realizadas de duas a três aferições da PA e, para fins de análise, foi considerada a última medida, desde que não houvesse diferença maior que $5 \mathrm{mmHg}$ entre as verificações. Caso isso ocorresse, eram realizadas mais duas medidas complementares, com intervalo mínimo de três minutos, considerando-se sempre a última medida.

O peso corporal foi medido em balança digital Tian2005D (Shan, China), com o avaliado trajando o mínimo possível de roupas, e a estatura medida com fita métrica inextensível posicionada em uma parede. $\mathrm{O}$ índice de massa corporal (IMC) foi calculado dividindo-se o peso pelo quadrado da estatura ${ }^{15}$.

Os dados foram analisados por meio dos softwares SPSS v.22 e GraphPad Prism 6.0, ambos para Windows. A normalidade da distribuição dos dados foi testada pelo método de D'Agostino-Pearson. As variáveis contínuas foram expressas como média \pm desvio padrão, e as categóricas em porcentagens. Comparações foram realizadas entre os normotensos e hipertensos, pelos testes de quiquadrado (proporções) ou teste T (médias). Em todos os casos, o nível de significância adotado foi de $5 \%$.

\section{RESULTADOS}

A Figura 1 ilustra as características de amostragem do presente estudo. Foram aleatoriamente selecionadas 409 pessoas entre os inscritos na UBS e, ao serem visitados no domicílio, no HR, foram encontrados 170 indivíduos (41,6\%) e, no HD, encontrados 239 (59,4\%). Dos 170 indivíduos do HR, 76 eram hipertensos (44,7\%, IC95\%: 37,2\%;52,5\%), sendo que, desses, 10 eram novos casos (13,2\%, IC95\%: 6,8\%;22,6\%). Dos 239 indivíduos encontrados no HD, 88 eram hipertensos (36,8\%, IC95\%: 30,8\%;43,3\%), sendo que, desses, 35 indivíduos 


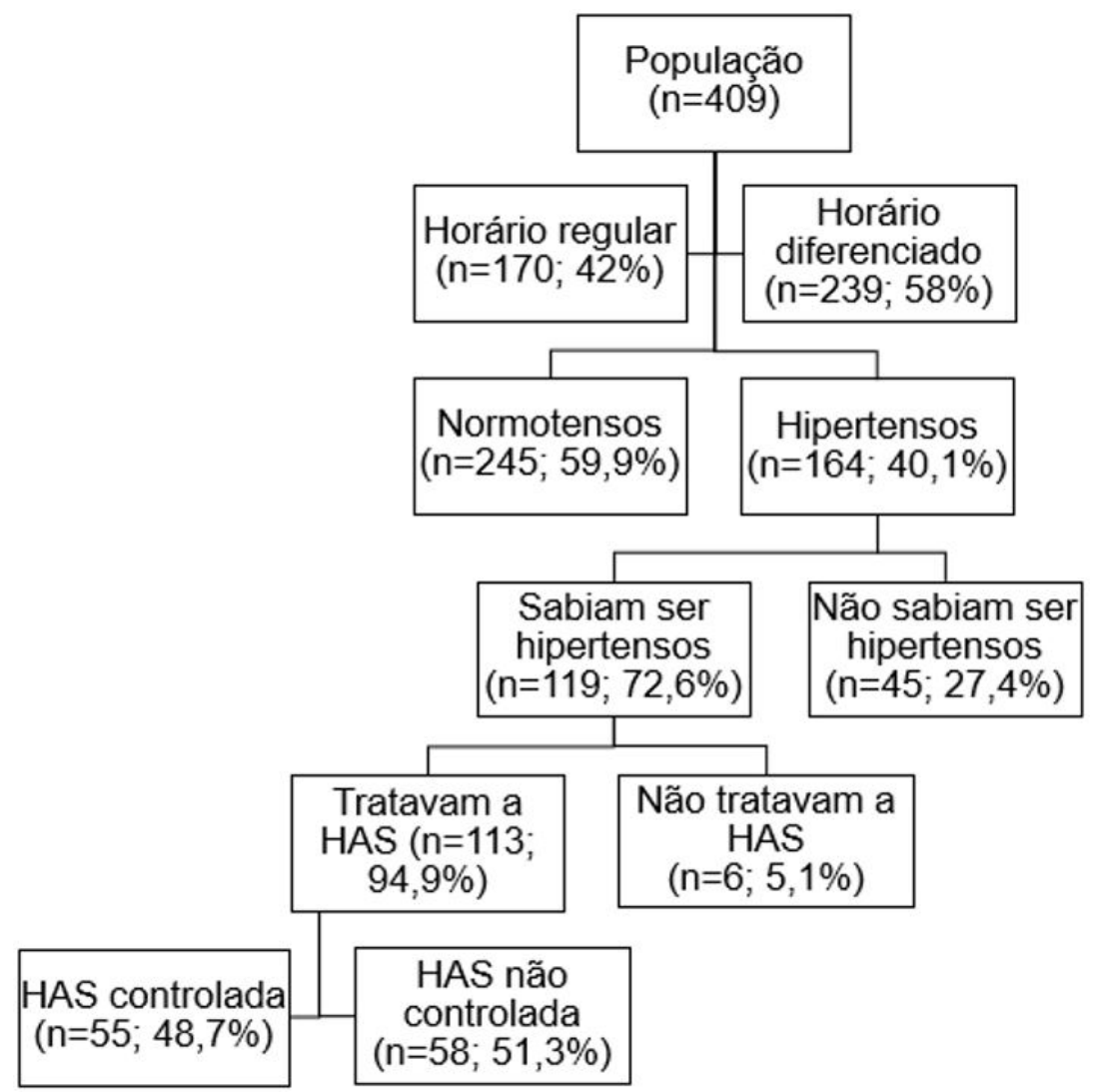

Figura 1. Prevalência de hipertensos segundo o grau de conhecimento, tratamento e controle. Unidade Básica de Saúde da região do Capão Redondo. São Paulo-SP, Brasil, 2015 (n=409)

(14,6\%, IC95\%: 29,7\%;50,8\%) eram novos casos de HAS. A prevalência de novos casos do HD foi significantemente maior que a do HR $(p<0,001)$. Assim sendo, se fosse calculada a prevalência de HAS, considerando-se somente os casos já conhecidos e os novos casos identificados no HR $(76+10=86$ casos de HAS do total de 409$)$, essa, provavelmente, seria de $21,0 \%$ (IC95\%: $17,3 \% ; 25,4 \%)$. Por outro lado, a prevalência de HAS, somando-se os casos conhecidos aos novos casos do HD, seria de $42,5 \%$ ( 76 conhecidos +10 novos +53 conhecidos +35 novos $=174$ casos de HAS do total de 409). Assim sendo, a prevalência de 42,5\% (IC95\%: 37,7\%;47,5\%), com a introdução do $H D$, elevaria significantemente $(p<0,001)$ a prevalência de HAS na população. Adicionalmente, admitindo-se que os 53 casos já conhecidos de HAS, encontrados no HD, fossem somados aos casos conhecidos e novos do HR $(86+53=139)$, a prevalência de HAS seria de apenas 33,9\% (IC95\%: 29,5\%;38,8\%), ainda assim, a proporção do HR seria significantemente inferior $(p=0,006)$ à proporção do HD. Esses resultados sugerem fortemente que calcular a prevalência de hipertensos com base somente no horário regular de funcionamento da UBS subestima a verdadeira prevalência de indivíduos com HAS.

A Tabela 1 resume os dados demográficos da amostra estudada. Participaram do estudo 409 indivíduos, sendo $31 \%$ do gênero masculino e $69 \%$ do gênero feminino, com média de idade de $48 \pm 17$ anos. Em relação ao IMC, 65\% ( $n=264)$ exibia excesso de peso (sobrepeso mais obesidade). Mais da metade da amostra (51\%) não havia completado o ensino médio, e a renda familiar per capita predominante foi de 1 a 2 salários mínimos (41\%).

Ao serem comparados normotensos e hipertensos (Tabela 2), os hipertensos foram significantemente mais velhos ( $p<0,001)$, apresentaram IMC significantemente maior $(p<0,001)$, maior proporção de analfabetos $(p<0,001)$ e com renda familiar menor que 2 salários mínimos $(p<0,001)$. Vinte e sete por cento $(27 \%)$ dos hipertensos desconheciam serem portadores de HAS e, entre os que sabiam serem hipertensos, $5 \%(n=6)$ não faziam tratamento. Entre os hipertensos em tratamento, $49 \%(n=55)$ apresentaram PA sob controle. 
Tabela 1. Características da população em relação às variáveis sociodemográficas. Unidade Básica de Saúde da região do Capão Redondo. São Paulo-SP, Brasil, 2015 (n=409)

\begin{tabular}{|c|c|c|c|c|}
\hline & & $\mathbf{n}$ & $\%$ & $\begin{array}{c}\text { Média } \pm \text { Desvio } \\
\text { Padrão }\end{array}$ \\
\hline \multirow[t]{2}{*}{ Gênero } & masculino & 127 & 31,1 & \\
\hline & feminino & 282 & 68,9 & \\
\hline Idade (anos) & & & & $48 \pm 2$ \\
\hline \multirow[t]{6}{*}{ Faixas Etárias } & 20 a 29 anos & 63 & $15,4 \%$ & \\
\hline & 30 a 39 anos & 75 & $18,3 \%$ & \\
\hline & 40 a 49 anos & 104 & $25,4 \%$ & \\
\hline & 50 a 59 anos & 60 & $14,7 \%$ & \\
\hline & 60 a 69 anos & 59 & $14,4 \%$ & \\
\hline & $>70$ anos & 48 & $11,7 \%$ & \\
\hline Peso $(k g)$ & & & & $73,2 \pm 16,9$ \\
\hline Estatura (cm) & & & & $162,8 \pm 9,6$ \\
\hline IMC $\left(\mathrm{kg} / \mathrm{m}^{2}\right)$ & & & & $27,6 \pm 5,9$ \\
\hline Classificação & Baixo peso & 18 & $4,4 \%$ & \\
\hline \multirow[t]{5}{*}{ do IMC } & Eutrófico & 127 & $31,1 \%$ & \\
\hline & Sobrepeso & 146 & $35,7 \%$ & \\
\hline & Obesidade grau I & 79 & $19,3 \%$ & \\
\hline & Obesidade grau II & 28 & $6,8 \%$ & \\
\hline & Obesidade grau III & 11 & $2,7 \%$ & \\
\hline \multirow[t]{5}{*}{ Escolaridade } & Analfabeto & 24 & $5,9 \%$ & \\
\hline & Até 40 ano & 102 & $24,9 \%$ & \\
\hline & EF completo & 82 & $20,0 \%$ & \\
\hline & EM completo & 154 & $37,7 \%$ & \\
\hline & ES completo & 47 & $11,5 \%$ & \\
\hline Renda & Até $1 / 2 \mathrm{SM}$ & 24 & 5,9 & \\
\hline \multirow[t]{4}{*}{ familiar } & $1 / 2$ a $1 \mathrm{SM}$ & 84 & 20,5 & \\
\hline & $1-2 \mathrm{SM}$ & 167 & 40,8 & \\
\hline & 2-4 SM & 87 & 21,2 & \\
\hline & $>4 \mathrm{SM}$ & 47 & 11,5 & \\
\hline
\end{tabular}

IMC: índice de massa corporal; EF: ensino fundamental ( $1^{\circ}$ ao $9^{\circ}$ ano); $\mathrm{EM}$ : ensino médio ( $10^{\circ}$ ao $12^{\circ}$ ano); $\mathrm{ES}$ (ensino superior / universitário); SM: salário mínimo $(\mathrm{R} \$ 905,00)$

\section{DISCUSSÃO}

O presente estudo buscou determinar a prevalência de indivíduos hipertensos e os níveis de conhecimento, tratamento e controle da doença, por meio de um método diferenciado. A prevalência de HAS encontrada foi muito superior à registrada anteriormente pelo método 
Tabela 2. Principais características da população hipertensa em relação aos normotensos. Unidade Básica de Saúde da região do Capão Redondo. São Paulo-SP, Brasil, 2015 ( $n=409)$

\begin{tabular}{|c|c|c|c|}
\hline & $\begin{array}{c}\text { Normotensos } \\
(n=245)\end{array}$ & Hipertensos $(n=164)$ & $\mathbf{p}$ \\
\hline Idade (anos)* & $40,6 \pm 13,7$ & $57,9 \pm 15,4$ & $<0.001^{\#}$ \\
\hline Maiores de 60 anos $^{* *}$ & 12,24 & 46,34 & $<0.001^{\# \#}$ \\
\hline Analfabetos & 2,44 & 10,98 & $<0.001^{\# \#}$ \\
\hline Escolaridade $<8$ anos & 40,81 & 45,13 & NS \\
\hline Renda familiar $\leq 2 \mathrm{SM}$ & 66,67 & 70,73 & $<0.001^{\# \#}$ \\
\hline IMC $\left(\mathrm{kg} / \mathrm{m}^{2)}\right.$ & $26,5 \pm 5,6$ & $29,2 \pm 6$ & $<0.001^{\#}$ \\
\hline Baixo peso ${ }^{* * *}$ & $15(6,1)$ & $3(1,8)$ & \\
\hline Eutrófico*** & $89(36,3)$ & $38(23,2)$ & \\
\hline Sobrepeso ${ }^{* * *}$ & $85(34,7)$ & $61(37,2)$ & \\
\hline Obesidade grau I*** & $39(15,9)$ & $40(24,4)$ & \\
\hline Obesidade grau II*** & $13(5,3)$ & $15(9,1)$ & \\
\hline Obesidade grau III*** & $4(1,6)$ & $7(4,3)$ & \\
\hline
\end{tabular}

*dados expressos como médias \pm desvios padrão; ${ }^{* *}$ dados expressos como proporções (\%); ${ }^{* * *}$ dados expressos como $\mathrm{n}$ (\%); "teste t; \#"quiquadrado; NS= diferença não significante. IMC: índice de massa corporal; SM: salário mínimo $(R \$ 905,00)$

tradicional ( $42,5 \%$ vs. $21 \%$, respectivamente). Também se verificou elevada prevalência de excesso de peso $(64,5 \%)$ na população estudada, bem superior aos valores de $54 \%$ a $56,9 \%$ encontrados entre brasileiros ${ }^{16,17}$.

Estudos de base populacional, envolvendo pessoas adultas e residentes em áreas urbanas, apresentam as seguintes prevalências de HAS: $23,2 \%{ }^{18} \mathrm{em}$ Sinop - AM, 25,2\% ${ }^{4} \mathrm{em}$ São José do Rio Preto - SP, 30,1\% ${ }^{19} \mathrm{em}$ Nobres - MT, $31,2 \%{ }^{20}$ no extremo oeste de Santa Catarina, $36,4 \%{ }^{21} \mathrm{em}$ Goiânia - GO e 40,5\%22 na cidade de Tubarão - SC. Adicionalmente, o IMC foi significantemente superior nos hipertensos que nos normotensos $(p<0,001)$. Estudos prévios já encontraram dados semelhantes, associando o excesso de peso à maior prevalência de HAS ${ }^{23,24}$. Supomos, portanto, que a elevada prevalência de excesso de peso no presente estudo esteja associada à elevada prevalência de HAS verificada.

Os hipertensos do presente estudo eram significantemente $(p<0,001)$ mais velhos que os indivíduos normotensos. Esse dado é apoiado pela literatura, que relata associação entre idade e a presença de HAS, pois o envelhecimento propicia alterações estruturais e funcionais no sistema cardiovascular que conduzem ao desenvolvimento da doença ${ }^{18}$.

A proporção de indivíduos analfabetos foi significantemente maior entre os hipertensos $(p<0,001)$ que entre os normotensos, fenômeno muito semelhante ao verificado por Rosário et al. ${ }^{19}$, na cidade de Nobres (MT), onde a prevalência de hipertensão foi 3 vezes maior em analfabetos quando comparados aos que possuíam 8 ou mais anos de escolaridade. Possivelmente, o menor conhecimento da patologia e a baixa assimilação das orientações gerais de prevenção, tratamento e controle da doença explicam a relação entre escolaridade e $\mathrm{HAS}^{25}$.

No presente estudo, observou-se proporção significantemente maior de indivíduos com renda familiar menor ou igual a 2 salários mínimos entre os hipertensos em relação aos normotensos $(70,7 \%$ vs. $66,6 \%, p<0,001)$. Achado semelhante foi encontrado por Weissheimer ${ }^{26}$ em uma amostra de 400 indivíduos da cidade de Cuiabá - MT. Pessoas em condições socioeconômicas menos favorecidas têm maior probabilidade de apresentar níveis pressóricos elevado ${ }^{27}$. A associação entre essas variáveis pode ser explicada pelo fato de a baixa renda potencializar os efeitos negativos de outros fatores como estresse psicossocial, hábitos alimentares inadequados, sedentarismo e aumento do peso corporal ${ }^{20}$. 
O percentual de conhecimento acerca de ser portador de HAS (73\%) se assemelhou ao encontrado por outro estudo brasileiro $\left(70 \%{ }^{19}\right)$, mas foi inferior ao verificado em população norte-americana $\left(83 \%{ }^{28}\right)$. A quantidade de hipertensos que aderiram ao tratamento, por outro lado, foi bastante superior no presente estudo (95\%), em relação ao verificado por Souza et al..$^{29}(54,3 \%)$, e ao observado na população dos Estados Unidos da América $(76 \%)^{28}$. Apesar disso, a proporção de hipertensos com controle pressórico verificada neste estudo se assemelhou à encontrada entre os hipertensos norte-americanos $\left(49 \%\right.$ vs. $\left.52 \%{ }^{28}\right)$. Outros estudos nacionais encontraram percentual de controle pressórico inferior ao observado neste estudo: $45,5 \%$ na região oeste da cidade de São Paulo ${ }^{30}, 43,1 \%$ em cidades do estado de Pernambuco ${ }^{31}$, e $33,7 \%$ em Novo Hamburgo (RS) ${ }^{29}$. Essas informações devem despertar maior preocupação em função dos agravos decorrentes da progressão da doença e de seus custos sociais, familiares e pessoais. Esses resultados possivelmente refletem as informações adequadas fornecidas à população sobre a doença, a participação de equipes multidisciplinares no sistema de saúde local e a disponibilização gratuita de medicamentos anti-hipertensivos, políticas estas também observadas em outros países em desenvolvimento ${ }^{32}$.

A maioria dos indivíduos randomizados $(58 \%, \mathrm{n}=239)$ foi encontrada no $\mathrm{HD}$ (aos domingos). O número de novos casos de hipertensão foi 3,5 vezes maior no $H D(n=35$ vs. $n=10$ ) em relação ao horário de funcionamento da UBS. Esse dado evidencia a dificuldade de encontrar a maioria das pessoas no domicílio no horário de funcionamento da UBS. O método diferenciado empregado neste estudo mostrou-se eficiente para reduzir a subnotificação de casos de HAS na UBS analisada, assim, sugere-se que ele seja replicado em grandes centros urbanos onde haja suspeita de subnotificação de casos de doenças crônicas.

O caráter transversal deste estudo representa uma limitação. Este tipo de desenho experimental impede o estabelecimento de associações causais entre a HAS e as demais características estudadas. Apesar disso, sinaliza a necessidade de novas formas de identificação de hipertensos, bem como de outras doenças.

O método diferenciado mostrou-se eficiente para reduzir a subnotificação de casos de HAS na população avaliada. A prevalência de HAS passou de $21 \%$ para $42,5 \%$. Entre os indivíduos hipertensos, $73 \%$ sabiam serem portadores da doença e, destes, $95 \%$ faziam tratamento. Entretanto, apenas $49 \%$ deles mantinham controle pressórico. Novos estudos que objetivem identificar as causas do baixo êxito no controle da PA são recomendados.

\section{REFERÊNCIAS}

1. Brasileira de Cardiologia S, Brasileira de Hipertensão S, Brasileira de Nefrologia S. VI Diretrizes Brasileiras de Hipertensão. Arq Bras Cardiol. 2010;95(1 Supl.):1-51. PMid:20694399.

2. Mills KT, Bundy JD, Kelly TN, Reed JE, Kearney PM, Reynolds K, et al. Global disparities of hypertension prevalence and control. Circulation. 2016;134(6):441-50. http://dx.doi.org/10.1161/ CIRCULATIONAHA.115.018912. PMid:27502908.

3. Picon RV, Fuchs FD, Moreira LB, Riegel G, Fuchs SC. Trends in prevalence of hypertension in Brazil: a systematic review with meta-analysis. PLoS One. 2012;7(10):e-48255. http://dx.doi.org/10.1371/journal. pone.0048255. PMid:23118964.

4. Cipullo JP, Martin FV, Ciorlia LAS, Godoy MRP, Cação JC, Loureiro AAC, et al. Prevalência e fatores de risco para hipertensão em uma população urbana brasileira. Arq Bras Cardiol. 2010;94(4):488-94. http://dx.doi. org/10.1590/S0066-782X2010005000014.

5. Jesus ES, Augusto MAO, Gusmão J, Mion D Jr, Ortega K, Pierin AMG. Profile of hypertensive patients: biosocial characteristics, knowledge, and treatment compliance. Acta Paul Enferm. 2008;21(1):59-65. http://dx.doi.org/10.1590/S0103-21002008000100009.

6. Mendes TAB, Goldbaum M, Segri NJ. Fatores associados à prevalência de práticas e controle de hipertensão entre os idosos residentes da cidade de São Paulo, Brasil. Cad Saude Publica. 2013;29(11):2275-86. http:// dx.doi.org/10.1590/0102-311x00151312. PMid:24233042.

7. Brasil. Ministério da Saúde. Secretaria de Atenção à Saúde. Departamento de Atenção Básica. Diretrizes do NASF: Núcleo de Apoio a Saúde da Família. Brasília: Ministério da Saúde, Secretaria de Atenção à Saúde, Departamento de Atenção Básica; 2010. 
8. Brasil. Ministério da Saúde. Secretaria de Atenção à Saúde. Departamento de Atenção Básica. Hipertensão arterial sistêmica para o Sistema Único de Saúde: Cadernos de Atenção Básica. Brasília: Ministério da Saúde; 2006.

9. Brasil. Ministério da Saúde. Departamento da Atenção Básica. Cobertura da Atenção Básica. [Internet] [citado em 2020 jul 1] Disponível em: https://egestorab.saude.gov.br/paginas/acessoPublico/relatorios/ relHistoricoCoberturaAB.xhtml

10. Brasil. Ministério da Saúde. Secretaria de Atenção à Saúde. Departamento de Atenção Básica. SIAB Sistema de Informação da Atenção Básica, dados coletados em nível municipal. Brasília: Ministério da Saúde; 2015.

11. Instituto Brasileiro de Geografia e Estatística. Censo demográfico 2010: características gerais da população - resultados da amostra. Rio de Janeiro; 2010.

12. Carril L. Quilombo, favela e periferia: a longa busca da cidadania. São Paulo: Annablume; Fapesp; 2006. $258 \mathrm{p}$.

13. Ferreira SRG, Moura EC, Malta DC, Sarno F. Frequência de hipertensão arterial e fatores associados: Brasil, 2006. Rev Saude Publica. 2009;43(Supl. 2):98-106. http://dx.doi.org/10.1590/S0034-89102009000900013. PMid:19936504.

14. Kearney PM, Whelton M, Reynolds K, Muntner P, Whelton PK, He J. Global burden of hypertension: analysis of worldwide data. Lancet. 2005;365(9455):217-23. http://dx.doi.org/10.1016/S0140-6736(05)17741-1. PMid:15652604.

15. World Health Organization. Obesity: preventing and managing the global epidemic. Reportof a WHO consultation on obesity. Geneva: WHO; 1997.

16. Louzada ML, Baraldi LG, Steele EM, Martins AP, Canella DS, Moubarac JC, et al. Consumption of ultraprocessed foods and obesity in Brazilian adolescentes and adults. Prev Med. 2015;81:9-15. http://dx.doi. org/10.1016/j.ypmed.2015.07.018. PMid:26231112.

17. Brasil. Ministério da Saúde. Instituto Brasileiro de Geografia e Estatística. Ministério do Planejamento, Orçamento e Gestão. Pesquisa Nacional de Saúde: 2013. Percepção do estado de saúde, estilos de vida e doenças crônicas. Brasil, grandes regiões e unidades da federação [Internet]. Rio de Janeiro: Instituto Brasileiro de Geografia e Estatística; 2014 [citado em 01 jul 2020]. Disponível em: http://189.28.128.100/ dab/docs/portaldab/documentos/PNS_Pesquisa_Nacional_de_Saude_2013_Volume_1.pdf.

18. Mikael LR, Paiva AMG, Gomes MM, Sousa ALL, Jardim PCBV, Vitorino PVO, et al. Vascular Aging and Arterial Stiffness. Arq Bras Cardiol. 2017;109(3):253-8. PMid:28678931.

19. Rosario TM, Scala LCN, França GVA, Pereira MRG, Jardim PCBV. Prevalência, controle e tratamento da hipertensão arterial sistêmica em Nobres - MT. Arq Bras Cardiol. 2009;93(6):672-8. http://dx.doi. org/10.1590/S0066-782X2009001200018.

20. De Sá CA, CorraloVD, Fachineto S, Schmidt CL, Cezar MA, Ribeiro CG. Obesidade, condição socioeconômica e hipertensão arterial no Extremo Oeste de Santa Catarina. Rev Salud Publica (Bogota). 2014;16(2):184-94. http://dx.doi.org/10.15446/rsap.v16n2.39536. PMid:25383493.

21. Jardim PCBV, Gondim MDRP, Monego ET, Moreira HG, Vitorino PVO, Souza WKSB, et al. Hipertensão arterial e alguns fatores de risco em uma capital brasileira. Arq Bras Cardiol. 2007;88(4):452-7. http:// dx.doi.org/10.1590/S0066-782X2007000400015. PMid:17546277.

22. Pereira MR, Coutinho MSSA, Freitas PF, D'Orsi E, Bernardi A, Hass R. Prevalência, conhecimento, tratamento e controle de hipertensão arterial sistêmica na população adulta urbana de Tubarão, Santa Catarina, Brasil, em 2003. Cad Saude Publica. 2007;23(10):2363-74. http://dx.doi.org/10.1590/S0102-311X2007001000011. PMid:17891297.

23. Portela PP, Mussi FC, Gama GGG, Santos CAST. Fatores associados ao descontrole da pressão arterial em homens. Acta Paul Enferm. 2016;29(3):307-15. http://dx.doi.org/10.1590/1982-0194201600043.

24. Moretti GS, Muniz PT, Carlos MT, Brunken GS, Farias JC Jr, Farias ES. Prevalência e fatores associados ao excesso de peso entre universitários de Rio Branco, Acre - Brasil. Rev Bras Cineantropom Desempenho Hum. 2014;16(4):406-18. http://dx.doi.org/10.5007/1980-0037.2014v16n4p406.

25. Andrade SSCA, Malta DC, Iser BM, Sampaio PC, Moura L. Prevalência da hipertensão arterial autorreferida nas capitais brasileiras em 2011 e análise de sua tendência no período de 2006 a 2011. Rev Bras Epidemiol. 2014;17(Supl. 1):215-26. http://dx.doi.org/10.1590/1809-4503201400050017. PMid:25054265.

26. Weissheimer FL. Incidência de hipertensão arterial em uma capital brasileira: estudo de base populacional. [dissertação]. Cuiabá, MT: Instituto de Saúde Coletiva da Universidade Federal de Mato Grosso; 2011. 
27. Taveira LF, Pierin AMG. Can the socioeconomic level influence the characteristics of a group of hypertensive patients? Rev Lat Am Enfermagem. 2007;15(5):929-35. http://dx.doi.org/10.1590/S010411692007000500008. PMid:18157444.

28. Nwankwo T, Yoon SS, Burt V, Gu Q. Hypertension among adults in the united states: national health and nutrition examination survey, 2011-2012. NCHS Data Brief. 2013;133(133):1-8. PMid:24171916.

29. Souza CS, Stein AT, Bastos GAN, Pellanda LC. Controle da pressão arterial em hipertensos do Programa Hiperdia: estudo de base territorial. Arq Bras Cardiol. 2014;102(6):571-8. PMid:25004419.

30. Pierin AMG, Marroni SN, Taveira LAF, Benseñor IJ. Controle da hipertensão arterial e fatores associados na atenção primária em Unidades Básicas de Saúde localizadas na Região Oeste da cidade de São Paulo. Cien Saude Colet. 2011;16(1 Suppl):1389-400. http://dx.doi.org/10.1590/S1413-81232011000700074. PMid:21503490.

31. Paes IMS. Estilo de vida e o controle da hipertensão arterial em indivíduos assistidos pela Estratégia de Saúde da Família, em Pernambuco. [dissertação]. Recife: Centro de Pesquisas Aggeu Magalhães da Fundação Oswaldo Cruz; 2012.

32. Orduñez-Garcia P, Munoz JL, Pedraza D, Espinosa-Brito A, Silva LC, Cooper RS. Success in control of hypertension in a low-resource setting: the cuban experience. J Hypertens. 2006;24(5):845-9. http:// dx.doi.org/10.1097/01.hjh.0000222753.67572.28. PMid:16612245. 